\title{
THE EXTREMES OF RANDOM WALKS IN RANDOM SCENERIES
}

\author{
BRICE FRANKE, ${ }^{*}$ Ruhr-Universität Bochum \\ TATSUHIKO SAIGO, ${ }^{* *}$ National Taiwan University
}

\begin{abstract}
In this article we analyse the behaviour of the extremes of a random walk in a random scenery. The random walk is assumed to be in the domain of attraction of a stable law, and the scenery is assumed to be in the domain of attraction of an extreme value distribution. The resulting random sequence is stationary and strongly dependent if the underlying random walk is recurrent. We prove a limit theorem for the extremes of the resulting stationary process. However, if the underlying random walk is recurrent, the limit distribution is not in the class of classical extreme value distributions.
\end{abstract}

Keywords: Extremes; stationary sequences; random walk; random scenery

2000 Mathematics Subject Classification: Primary 60G70; 60G50

\section{Introduction}

Extreme value theory has been developed for independent, identically distributed (i.i.d.) random variables by Gnedenko in the 1940s (see Gnedenko (1943)). He proved that those maxima converge toward special types of random variables called extreme value distributions. Later, these theorems were generalised to stationary sequences of dependent random variables. In order to address these problems, some concepts of fading dependence were introduced. Among the most advanced of these concepts were the $D\left(u_{n}\right)$ and $D^{\prime}\left(u_{n}\right)$ conditions (see Section 3), which were introduced by Leadbetter to describe a weak-mixing-type dependence for the tails of stationary sequences. If they are satisfied, the random sequence behaves essentially like the independent sequence with the same individual distributions (see Leadbetter et al. (1983, p. 57)). Another concept which quantifies the amount of clustering in the dependent sequence is the extremal index. If the extremal index is larger than 0 , the asymptotics of the extremes for the dependent sequence can be compared to the independent situation (see Leadbetter et al. (1983, p. 68)).

In this article we investigate the behaviour of a special dependent stationary sequence. Specifically, we investigate the behaviour of a random walk in a random scenery, where the random walk is assumed to be in the domain of attraction of a stable Lévy process and the scenery consists of random variables which are in the domain of attraction of an extreme value distribution. We will see that if the underlying random walk is recurrent, the sequence does not satisfy the $D\left(u_{n}\right)$ condition introduced by Leadbetter. However, we can prove a limit theorem in this situation. In the transient case, the resulting sequence satisfies the $D\left(u_{n}\right)$ condition, but not the $D^{\prime}\left(u_{n}\right)$ condition. We also compute the extremal index for a random walk in a random

Received 21 August 2007; revision received 28 October 2008.

* Postal address: Ruhr-Universität Bochum, Fakultät für Mathematik, Universitätsstr. 150, 44780 Bochum, Germany. Email address: brice.franke@rub.de

** Current address: Department of Mathematics, Keio University 3-14-1 Hiyoshi, Kohoku-ku, Yokohama-shi, Kanagawa-ken prefecture, 223-8522, Japan. Email address: saigo@math.keio.ac.jp 
scenery. It turns out to be 0 when the natural scaling of the underlying random walk is larger than 1.

Our investigation is motivated by the work of Kesten and Spitzer (1979). They proved limit theorems for the sums of stationary sequences arising from recurrent random walks in random sceneries. The resulting limits turned out to be new kinds of self-similar processes with strong dependence in the recurrent case.

We now come to the definition of the model that we will investigate in this article. Let $\left\{X_{k}, k \in \mathbb{N}\right\}$ be a sequence of centred, integer-valued, i.i.d. random variables with the property that, for $S_{n}=X_{1}+\cdots+X_{n}$ and all $x \in \mathbb{R}$, we have

$$
\mathrm{P}\left(n^{-1 / \alpha} S_{n} \leq x\right) \rightarrow F_{\alpha}(x) \text { as } n \rightarrow \infty,
$$

where $F_{\alpha}$ is the distribution function of a stable law with characteristic function given by

$$
\varphi(\theta)=\exp \left(-|\theta|^{\alpha}\left(C_{1}+\mathrm{i} C_{2} \operatorname{sgn} \theta\right)\right), \quad \alpha \in(0,2] .
$$

We will denote by $\{Y(t), t \geq 0\}$ the right-continuous $\alpha$-stable Lévy process with characteristic function given by $\varphi(t \theta)$. It is well known that the processes

$$
S^{(n)}(t):=n^{-1 / \alpha} S_{[n t]}
$$

converge in distribution to $Y$ with respect to the Skorokhod topology (see Kesten and Spitzer (1979)). Here and in the following we denote by $[x]$ the integer part of a positive real number $x$.

Let $\{\xi(k), k \in \mathbb{Z}\}$ be a family of $\mathbb{R}$-valued, i.i.d. random variables which we assume to be independent of the sequence $\left\{X_{k}, k \in \mathbb{N}\right\}$. The sequence of random variables $\left\{\xi\left(S_{n}\right), n \in \mathbb{N}\right\}$ is called a random walk in a random scenery in the literature. The sequence $\left\{\xi\left(S_{n}\right), n \in \mathbb{N}\right\}$ is a stationary sequence with some nontrivial long-range dependence.

For the random variables $\{\xi(n), n \in \mathbb{Z}\}$ belonging to the domain of attraction of a stable law with exponent $\beta$ and for $\alpha \neq 1$, Kesten and Spitzer (1979) proved a limit theorem for the sum

$$
W_{n}:=\xi\left(S_{1}\right)+\cdots+\xi\left(S_{n}\right)
$$

It turns out that the scaled sequence $W^{(n)}(t):=n^{-\delta} W_{[n t]}$ converges in distribution to a selfsimilar process. The scaling exponent $\delta$ is $1-1 / \alpha+1 / \alpha \beta$ if $\alpha \in(1,2]$ and $\beta$ if $\alpha \in(0,1)$. The case in which $\alpha=1$ is more difficult, since the underlying random walk is then only zero-recurrent. Besides the fundamental work of Kesten and Spitzer, a lot of refinements and generalisations in various directions were obtained by other authors (see Lang and Nguyen (1983), Shieh (1995), Maejima (1996), Arai (2001), and Saigo and Takahashi (2005)).

In this article we investigate the asymptotic behaviour of the maxima

$$
K_{n}:=\max \left\{\xi\left(S_{1}\right), \xi\left(S_{2}\right), \ldots, \xi\left(S_{n}\right)\right\} .
$$

For this, we will assume that the distribution function $F$ of the random variables $\{\xi(n), n \in \mathbb{Z}\}$ is in the domain of attraction of an extreme value distribution $G(x)$. This means that there exist two sequences $\left\{a_{n}>0, n \in \mathbb{N}\right\}$ and $\left\{b_{n} \in \mathbb{R}, n \in \mathbb{N}\right\}$ such that, for

$$
M_{n}:=\max \{\xi(1), \ldots, \xi(n)\},
$$

we have

$$
\mathrm{P}\left(\frac{M_{n}-b_{n}}{a_{n}} \leq x\right)=\left(F\left(a_{n} x+b_{n}\right)\right)^{n} \rightarrow G(x) \quad \text { as } n \rightarrow \infty .
$$


The convergence to types theorem reduces the class of possible limit distributions $G$ to three particular cases: the Fréchet distribution, the Weibull distribution, and the Gumbel distribution (see Resnick (1987, p. 9)). The corresponding domains of attraction are well known and can be found in Resnick (1987, p. 38ff).

To the distribution function $G$, we associate an extreme value process having finitedimensional distributions defined as

$$
G_{t_{1}, \ldots, t_{k}}\left(x_{1}, \ldots, x_{k}\right):=G^{t_{1}}\left(\bigwedge_{i=1}^{k} x_{i}\right) G^{t_{2}-t_{1}}\left(\bigwedge_{i=2}^{k} x_{i}\right) \cdots G^{t_{k}-t_{k-1}}\left(x_{k}\right) .
$$

The resulting stochastic process $\{Z(t), t>0\}$ is a Markov process with nondecreasing paths. A version of this process exists in $D(0, \infty)$. We define the sequence

$$
Z^{(n)}(t):=\frac{M_{[n t]}-b_{n}}{a_{n}} .
$$

It is well known that $Z^{(n)}$ converges in distribution to $Z$ with respect to the Skorokhod topology (see Resnick (1987, p. 211)).

The difference between the sequence $M_{n}=\max \{\xi(1), \ldots, \xi(n)\}$ and the sequence $K_{n}=$ $\max \left\{\xi\left(S_{1}\right), \ldots, \xi\left(S_{n}\right)\right\}$ is due to the fact that the random walk $\left\{S_{n}, n \in \mathbb{N}\right\}$ visits certain sites several times. It is obvious that the distribution of $K_{n}$ depends on the number of sites that the random walk $\left\{S_{k}, k \in \mathbb{N}\right\}$ has visited until time $n$. In fact, it is the range

$$
R_{n}:=\operatorname{card}\left\{S_{1}, \ldots, S_{n}\right\}
$$

of the underlying random walk $\left\{S_{k}, k \in \mathbb{N}\right\}$ which determines the behaviour of the sequence $\left\{K_{n}, n \in \mathbb{N}\right\}$. The asymptotic behaviour of the range for the rescaled integer-valued random walks $S^{(n)}$ defined in (1) can be found in Le Gall and Rosen (1991). They presented the following results. If $S^{(n)}$ converges to an $\alpha$-stable Lévy process $Y$ then the following statements hold.

(R1) If $\alpha<1$ then we have

$$
\frac{1}{n} R_{[n t]} \rightarrow q t \quad \mathrm{P} \text {-almost surely as } n \rightarrow \infty,
$$

where $q:=\mathrm{P}\left(S_{k} \neq 0, k \in \mathbb{N}\right)$.

(R2) If $\alpha=1$ then we have

$$
\frac{h(n)}{n} R_{[n t]} \rightarrow t \quad \text { in } L^{p}(\Omega, \mathrm{P}) \text { as } n \rightarrow \infty,
$$

where

$$
h(n):=1+\sum_{k=1}^{n} \mathrm{P}\left(S_{k}=0\right)
$$

is the truncated Green function.

(R3) If $1<\alpha \leq 2$ then we have, for all $L \in \mathbb{N}$ and all $t_{1}<t_{2}<\cdots<t_{L}$,

$$
n^{-1 / \alpha}\left(R_{\left[n t_{1}\right]}, \ldots, R_{\left[n t_{L}\right]}\right) \rightarrow\left(m\left(Y\left(0, t_{1}\right)\right), \ldots, m\left(Y\left(0, t_{L}\right)\right)\right)
$$

in distribution as $n \rightarrow \infty$, where $m$ denotes the Lebesgue measure on $\mathbb{R}$. 
Remark. The first statement was proved by Kesten, Spitzer, and Whitman for $0<\alpha \leq 2$. However, $q$ is equal to 0 in the transient case, i.e. $\alpha>1$ (see Spitzer (1976, p. 35ff)). For $\frac{2}{3} \leq \alpha<1$, we know that $h(n) \rightarrow q^{-1}$ as $n \rightarrow \infty$, and (R1) yields the almost-sure convergence of $(h(n) / n) R_{[n t]}$ towards $t$ (see Le Gall and Rosen (1991)). The case in which $\alpha=1$ is a particular case since in this situation it is in general not known whether the random walk is transient or recurrent. The second and third statements were proved in Le Gall and Rosen (1991). We mention that Le Gall and Rosen (1991) only stated the marginal convergence in (R3). However, their proof also covered the joined convergence described in (R3). The reason is as follows. To prove the convergence in distribution of $R_{[n t]}$ towards $m(Y(0, t))$, they used the Skorokhod representation theorem to introduce a process $\left\{\tilde{S}^{(n)}(t), t \geq 0\right\}$ which has the same distribution as $\left\{S^{(n)}(t), t \geq 0\right\}$ and converges almost surely to $\{Y(t), t \geq 0\}$ with respect to the Skorokhod topology. For all $n \in \mathbb{N}$, the associated range processes $\left\{\overline{\tilde{R}}_{t}^{(n)}, t \geq 0\right\}$ then have the same distributions as $\left\{R_{[n t]}, t \geq 0\right\}$. Le Gall and Rosen then proved that $n^{-1 / \alpha} \tilde{R}_{t}^{(n)}$ converge in $L^{1}$ to $m(Y(0, t))$ for all $t \geq 0$. This also implies the $L^{1}$-convergence of the vectors $n^{-1 / \alpha}\left(\tilde{R}_{t_{1}}^{(n)}, \ldots, \tilde{R}_{t_{L}}^{(n)}\right)$ towards the vector $\left(m\left(Y\left(0, t_{1}\right)\right), \ldots, m\left(Y\left(0, t_{L}\right)\right)\right)$, which yields (R3).

We are now in the position to state the first main result.

Theorem 1. For $\alpha \leq 1$, the sequence

$$
K^{(n)}(t):=\frac{\max \left\{\xi\left(S_{1}\right), \ldots, \xi\left(S_{[n t]}\right)\right\}-b_{m(n)}}{a_{m(n)}}
$$

converges in distribution to the extreme value process $Z$ associated to the extreme value distribution $G$, where

$$
m(n):= \begin{cases}{[q n]} & \text { for } \alpha<1, \\ {\left[\frac{n}{h(n)}\right]} & \text { for } \alpha=1 .\end{cases}
$$

This theorem is a classical result in the sense that the limit distribution is again an extreme value distribution. Only the scaling has to be modified according to the behaviour of the range of the underlying random walk. We will see in the final section that the sequence $\left\{\xi\left(S_{n}\right), n \in \mathbb{N}\right\}$ satisfies the $D\left(u_{n}\right)$ condition, but not the $D^{\prime}\left(u_{n}\right)$ condition for appropriate sequences $\left\{u_{n}, n \in \mathbb{N}\right\}$ when $\alpha<1$. Furthermore, we will see in the final section that the extremal index of the sequence $\left\{\xi\left(S_{n}\right), n \in \mathbb{N}\right\}$ can be computed and is equal to $q$. However, $q$ turns out to be 0 for $\alpha>1$. This explains why we have to modify the scaling in the second statement. Subsequently, we will see that, for $\alpha>1$, the sequence $\left\{\xi\left(S_{n}\right), n \in \mathbb{N}\right\}$ does not satisfy the $D\left(u_{n}\right)$ condition for the sequence $u_{n}:=a_{\left[n^{1 / \alpha}\right]} x+b_{\left[n^{1 / \alpha}\right]}$. However, we can prove the following limit theorem.

Theorem 2. For $\alpha>1$, the sequence

$$
K_{t}^{(n)}:=\frac{\max \left\{\xi\left(S_{1}\right), \ldots, \xi\left(S_{[n t]}\right)\right\}-b_{\left[n^{1 / \alpha}\right]}}{a_{\left[n^{1 / \alpha}\right]}}
$$

converges in distribution to the stochastic process

$$
K(t):=Z(m(Y(0, t))) .
$$

It is important to note that the limit distribution in Theorem 2 is not of extreme value type. We will discuss this in the final section of this article. 
The stationary sequence $\left\{\xi\left(S_{n}\right), n \in \mathbb{N}\right\}$ is dependent due to the recurrence of an underlying random walk. There have been some investigations on stationary sequences with dependence resulting from an underlying Markov structure. The most popular of these concepts is the chaindependent sequence, which has been studied extensively in extreme value theory (see Resnick (1972), Denzel and O'Brien (1975), and Turkman and Oliveira (1992)). However, we will see in the final section that the sequence $\left\{\xi\left(S_{n}\right), n \in \mathbb{N}\right\}$ is not chain dependent. A generalisation of chain dependence has been introduced in Turkman and Walker (1983). Nevertheless, the underlying process in their model has only a finite state space and an invariant measure, which is not the case for the integer-valued random walk studied in this article.

\section{Proof of the limit theorems}

In many extreme value situations there exists an underlying point process. Often it is more suitable to prove limit theorems on the level of those point processes and then to use the continuous mapping theorem in order to understand the behaviour of the extremes. We will follow this approach in our subsequent investigation. We first define the stopping times

$$
\tau_{k}:=\inf \left\{m \in \mathbb{N} ; \operatorname{card}\left\{S_{1}, \ldots, S_{m}\right\} \geq k\right\},
$$

and note that

$$
K_{n}=\max \left\{\xi\left(S_{\tau_{k}}\right) ; \tau_{k} \leq n\right\}
$$

Moreover, we define

$$
Q^{(n)}\left(t_{1}, t_{2}\right]:=\operatorname{card}\left\{m \in \mathbb{N} ; n t_{1}<m \leq n t_{2}, S_{m} \notin\left\{S_{1}, \ldots, S_{m-1}\right\}\right\} .
$$

The process $S^{(n)}$ defined in (1) visits a new site during the time interval $\left(t_{1}, t_{2}\right]$ if and only if there exists an integer $k$ such that $\tau_{k} / n \in\left(t_{1}, t_{2}\right]$. This implies that the total number of new sites visited by $S^{(n)}$ during the time interval $\left(t_{1}, t_{2}\right]$ is $\sum_{k} \mathbf{1}_{\left(t_{1}, t_{2}\right]}\left(\tau_{k} / n\right)$. We therefore have the following identity:

$$
Q^{(n)}\left(t_{1}, t_{2}\right]=\sum_{k} \mathbf{1}_{\left(t_{1}, t_{2}\right]}\left(\frac{\tau_{k}}{n}\right)=R_{\left[n t_{2}\right]}-R_{\left[n t_{1}\right]} .
$$

The next lemma states the independence of the $\xi\left(S_{k}\right)$-sequence and the $\tau_{k}$-sequence.

Lemma 1. For all $L \in \mathbb{N}$, and all measurable sets $B_{k} \subset \mathbb{N}$ and $A_{k} \subset \mathbb{R}$ with $1 \leq k \leq L$, we have

$$
\begin{aligned}
\mathrm{P}\left(\tau_{k}\right. & \left.\in B_{k}, \xi\left(S_{\tau_{k}}\right) \in A_{k}, 1 \leq k \leq L\right) \\
& =\mathrm{P}\left(\xi(k) \in A_{k}, 1 \leq k \leq L\right) \mathrm{P}\left(\tau_{k} \in B_{k}, 1 \leq k \leq L\right) .
\end{aligned}
$$

Proof. We use the independence of the random walk and the scenery to prove that

$$
\begin{aligned}
\mathrm{P}\left(\tau_{k} \in B_{k}, \xi\left(S_{\tau_{k}}\right) \in A_{k}\right) & =\sum_{m \in B_{k}} \mathrm{P}\left(\tau_{k}=m, \xi\left(S_{m}\right) \in A_{k}\right) \\
& =\sum_{m \in B_{k}} \sum_{z \in \mathbb{Z}} \mathrm{P}\left(\tau_{k}=m, S_{m}=z, \xi(z) \in A_{k}\right) \\
& =\sum_{m \in B_{k}} \sum_{z \in \mathbb{Z}} \mathrm{P}\left(\tau_{k}=m, S_{m}=z\right) \mathrm{P}\left(\xi(k) \in A_{k}\right) \\
& =\mathrm{P}\left(\tau_{k} \in B_{k}\right) \mathrm{P}\left(\xi(k) \in A_{k}\right) .
\end{aligned}
$$

The general case follows by a similar proof. 
For an $L \in \mathbb{N}$, we denote by $\mathrm{P}_{\xi}$ the joint distribution of $\{\xi(k), 1 \leq k \leq L\}$ on $\mathbb{R}^{L}$.

Lemma 2. For every $L \in \mathbb{N}$ and every bounded measurable function

$$
f: \mathbb{R}^{L} \times \mathbb{N}^{L} \rightarrow \mathbb{R}, \quad\left(\left(x_{k}\right),\left(m_{k}\right)\right) \mapsto f\left(\left(x_{k}\right),\left(m_{k}\right)\right),
$$

we have

$$
\mathrm{E}\left[f\left(\left(\xi\left(S_{\tau_{k}}\right)\right),\left(\tau_{k}\right)\right)\right]=\mathrm{E}\left[\int_{\mathbb{R}^{L}} f\left(\left(x_{k}\right),\left(\tau_{k}\right)\right) \mathrm{P}_{\xi}\left(\mathrm{d}\left(x_{k}\right)\right)\right] .
$$

Proof. In order to avoid notational overload, we just prove a simplified statement for the one-dimensional marginal distributions. Let

$$
f(x, m)=\sum_{i=1}^{M} \sum_{j=1}^{K} \alpha_{i j} \mathbf{1}_{A_{i}}(x) \mathbf{1}_{B_{j}}(m)
$$

be a step function over measurable sets $A_{i}, 1 \leq i \leq M$, in $\mathbb{R}$ and $B_{j}, 1 \leq j \leq K$, in $\mathbb{N}$. Then it follows from the previous lemma that

$$
\begin{aligned}
\mathrm{E}\left[f\left(\xi\left(S_{\tau_{k}}\right), \tau_{k}\right)\right] & =\sum_{i=1}^{M} \sum_{j=1}^{K} \alpha_{i j} \mathrm{P}\left(\xi\left(S_{\tau_{k}}\right) \in A_{i}, \tau_{k} \in B_{j}\right) \\
& =\sum_{i=1}^{M} \sum_{j=1}^{K} \alpha_{i j} \mathrm{P}\left(\xi(k) \in A_{i}\right) \mathrm{P}\left(\tau_{k} \in B_{j}\right) \\
& =\mathrm{E}\left[\int_{\mathbb{R}} f\left(x, \tau_{k}\right) \mathrm{P}_{\xi(k)}(\mathrm{d} x)\right] .
\end{aligned}
$$

The result now follows from a monotone class argument.

Now we use the sequence $\left\{\xi\left(S_{\tau_{k}}\right), k \in \mathbb{N}\right\}$ to construct a sequence of random measures on a suitable state space. We denote by $P_{n}$ the distribution of the random variable $\left(\xi(1)-b_{n}\right) / a_{n}$. If $\xi(1)$ is in the domain of attraction of the extreme value distribution $G$, it is well known that

$$
n P_{n}((x, \infty))=n \mathrm{P}\left(\frac{\xi(1)-b_{n}}{a_{n}}>x\right) \rightarrow-\log (G(x)) \quad \text { as } n \rightarrow \infty .
$$

This can be rephrased as the vague convergence of $n P_{n}$ towards a suitable measure $v$ on a suitable topological space $E$. If $G$ is a Fréchet distribution, this holds for the right-compactified interval $E:=(0, \infty]$ and $\nu(x, \infty]:=x^{-\gamma}$; if $G$ is a Weibull distribution, this holds for $E:=(-\infty, 0]$ and the measure $v(x, 0]:=(-x)^{-\gamma}$; if $G$ is a Gumbel distribution, this holds for the rightcompactified interval $E:=(-\infty, \infty]$ and $\nu(x, \infty]:=\mathrm{e}^{-x}$ (see Resnick (1987, p. 210)).

For a Borel measure $\kappa$ on $\mathbb{R}^{+} \times E$ and a measurable function $f: \mathbb{R}^{+} \times E \rightarrow[0, \infty)$, we define

$$
\kappa(f):=\int_{\mathbb{R}^{+}} \int_{E} f(s, x) \kappa(\mathrm{d} s, \mathrm{~d} x) .
$$

To the intensity measure $\mu:=m \times v$ on $\mathbb{R}^{+} \times E$, there exists a Poisson point process $N$ which is characterised by its Laplace functional through

$$
\mathrm{E}\left[\mathrm{e}^{-N(f)}\right]=\exp \left(-\int_{\mathbb{R}^{+}} \int_{E}\left(1-\mathrm{e}^{f(s, x)}\right) \nu(\mathrm{d} x) \mathrm{d} s\right) .
$$


We denote by $\mathcal{M}_{p}$ the set of point measures on $\mathbb{R}^{+} \times E$, and remark that $\mathcal{M}_{p}$ is a closed subset of the set $\mathcal{M}_{+}$of Borel measures with respect to the vague topology (see Resnick (1987, p. 140)). We are now in position to state the theorem for the transient situation.

Theorem 3. For $\alpha \leq 1$, the point processes

$$
N^{(n)}:=\sum_{k} \delta_{\left(\tau_{k} / n,\left(\xi\left(S_{\tau_{k}}\right)-b_{m(n)}\right) / a_{m(n)}\right)}
$$

converge weakly to the Poisson point process $N$ with intensity measure $m \times v$, where

$$
m(n):= \begin{cases}{[q n]} & \text { for } \alpha<1, \\ {\left[\frac{n}{h(n)}\right]} & \text { for } \alpha=1 .\end{cases}
$$

In the following, let $N_{Y}$ be the Poisson point process on $\mathbb{R}^{+} \times E$ with random intensity measure

$$
\mu(\mathrm{d} t, \mathrm{~d} x)=m_{Y}(\mathrm{~d} t) \times v(\mathrm{~d} x),
$$

where $m_{Y}(t):=m(Y(0, t))$ is a random distribution function on $\mathbb{R}^{+}$. Such point processes are called Cox processes in the literature (see Daley and Vere-Jones (1988, p. 261)). For an arbitrary continuous function $f: \mathbb{R}^{+} \times E \rightarrow \mathbb{R}$ with compact support, the Laplace functional of $N_{Y}$ is given by

$$
\begin{aligned}
\mathcal{L}(f) & :=\mathrm{E}\left[\exp \left(-N_{Y}(f)\right)\right] \\
& =\mathrm{E}\left[\exp \left(-\int_{\mathbb{R}^{+}} \int_{E}\left(1-\mathrm{e}^{-f(s, x)}\right) \nu(\mathrm{d} x) m_{Y}(\mathrm{~d} s)\right)\right] .
\end{aligned}
$$

Theorem 4. If we define $\tilde{a}_{n}:=a_{\left[n^{1 / \alpha}\right]}$ and $\tilde{b}_{n}:=b_{\left[n^{1 / \alpha}\right]}$, the point processes

$$
N^{(n)}:=\sum_{k} \delta_{\left(\tau_{k} / n,\left(\xi\left(S_{\tau_{k}}\right)-\tilde{b}_{n}\right) / \tilde{a}_{n}\right)}
$$

on $\mathbb{R}^{+} \times E$ converge weakly to the point process $N_{Y}$.

Proof. We give a detailed proof of Theorem 4; however, a proof of Theorem 3 can be obtained from this by changing the scale parameters and by using (R1) and (R2), respectively, instead of (R3) in the subsequent proof.

The Laplace functional of $N^{(n)}$ is given by

$$
\begin{aligned}
\mathscr{L}_{n}(f) & =\mathrm{E}\left[\exp \left(-N^{(n)}(f)\right)\right] \\
& =\mathrm{E}\left[\exp \left(-\sum_{k} f\left(\frac{\tau_{k}}{n}, \frac{\xi\left(S_{\tau_{k}}\right)-\tilde{b}_{n}}{\tilde{a}_{n}}\right)\right)\right] .
\end{aligned}
$$

We have to prove that the Laplace functionals of $N^{(n)}$ converge to the Laplace functional of $N_{Y}$ (see Resnick (1987, p. 153)).

Let $f: \mathbb{R}^{+} \times E \rightarrow[0, \infty)$ be a continuous function with compact support contained in $\left(t_{0}, T\right] \times E$. For $L \in \mathbb{N}$, let $\left\{0<t_{0}<\cdots<t_{L}=T\right\}$ be a partition with the property that $t_{i+1}-t_{i}<1 / m$. We can define the following truncated function:

$$
f_{m}(t, x):=\sum_{i=0}^{L-1} \mathbf{1}_{\left(t_{i}, t_{i+1}\right]}(t) g_{i}(x),
$$


where $g_{i}(x):=\inf _{t_{i} \leq s \leq t_{i+1}} f(s, x)$. We know that $f_{m} \uparrow f$ uniformly in $\mathbb{R}^{+} \times E$ as $m \rightarrow \infty$. The Laplace functional in $f_{m}$ has the form

$$
\begin{aligned}
\mathscr{L}_{n}\left(f_{m}\right) & =\mathrm{E}\left[\exp \left(-\sum_{k} f_{m}\left(\frac{\tau_{k}}{n}, \frac{\xi\left(S_{\tau_{k}}\right)-\tilde{b}_{n}}{\tilde{a}_{n}}\right)\right)\right] \\
& =\mathrm{E}\left[\exp \left(-\sum_{k} \sum_{i} g_{i}\left(\frac{\xi\left(S_{\tau_{k}}\right)-\tilde{b}_{n}}{\tilde{a}_{n}}\right) \mathbf{1}_{\left(t_{i}, t_{i+1}\right]}\left(\frac{\tau_{k}}{n}\right)\right)\right] .
\end{aligned}
$$

We apply Lemma 2 and obtain

$$
\mathcal{L}_{n}\left(f_{m}\right)=\mathrm{E}\left[\int_{\mathbb{R}^{L}} \exp \left(-\sum_{k} \sum_{i} g_{i}\left(\frac{x_{k}-\tilde{b}_{n}}{\tilde{a}_{n}}\right) \mathbf{1}_{\left(t_{i}, t_{i+1}\right]}\left(\frac{\tau_{k}}{n}\right)\right) \mathrm{P}_{\xi}\left(\mathrm{d}\left(x_{k}\right)\right)\right] .
$$

Now, we can use the i.i.d. property of the sequence $\{\xi(k), k \in \mathbb{N}\}$ to obtain

$$
\begin{aligned}
\mathcal{L}_{n}\left(f_{m}\right) & =\mathrm{E}\left[\prod_{k} \int_{E} \exp \left(-\sum_{i} g_{i}\left(\frac{x-\tilde{b}_{n}}{\tilde{a}_{n}}\right) \mathbf{1}_{\left(t_{i}, t_{i+1}\right]}\left(\frac{\tau_{k}}{n}\right)\right) \mathrm{P}_{\xi(1)}(\mathrm{d} x)\right] \\
& =\mathrm{E}\left[\prod_{k} \prod_{i}\left(1-\int_{E}\left(1-\exp \left(-g_{i}(z) \mathbf{1}_{\left(t_{i}, t_{i+1}\right]}\left(\frac{\tau_{k}}{n}\right)\right)\right) P_{\left[n^{1 / \alpha}\right]}(\mathrm{d} z)\right)\right] .
\end{aligned}
$$

For the following, we define

$$
\lambda_{i, k ; n}:=\int_{E} \exp \left(-g_{i}(z) \mathbf{1}_{\left(t_{i}, t_{i+1}\right]}\left(\frac{\tau_{k}}{n}\right)\right) P_{\left[n^{1 / \alpha}\right]}(\mathrm{d} z)
$$

and

$$
\varphi_{i, k ; n}:=\exp \left(-n^{-1 / \alpha} \int_{E}\left(1-\exp \left(-g_{i}(z) \mathbf{1}_{\left(t_{i}, t_{i+1}\right]}\left(\frac{\tau_{k}}{n}\right)\right)\right) v(\mathrm{~d} z)\right) .
$$

As $n P_{n}$ converges to $v$ in the vague topology, we have, uniformly for all $1 \leq i \leq L$ and all $k \in \mathbb{N}$,

$$
-\left(1-\lambda_{i, k ; n}\right) \sim \log \left(\varphi_{i, k ; n}\right) \quad \text { as } n \rightarrow \infty .
$$

This implies that

$$
\log \left(\lambda_{i, k ; n}\right) \sim \log \left(\varphi_{i, k ; n}\right) \quad \text { as } n \rightarrow \infty .
$$

From this we deduce that

$$
\sup _{i, k}\left|\frac{\log \left(\lambda_{i, k ; n}\right)-\log \left(\varphi_{i, k ; n}\right)}{\log \left(\varphi_{i, k ; n}\right)}\right| \rightarrow 0 \quad \text { as } n \rightarrow \infty .
$$

We note that

$$
\begin{aligned}
& \left|\sum_{i, k} \log \left(\lambda_{i, k ; n}\right)-\sum_{i, k} \log \left(\varphi_{i, k ; n}\right)\right| \\
& \quad \leq R_{[n T]} \sup _{i, k}\left|\log \left(\varphi_{i, k ; n}\right)\right| \sup _{i, k}\left|\frac{\log \left(\lambda_{i, k ; n}\right)-\log \left(\varphi_{i, k ; n}\right)}{\log \left(\varphi_{i, k ; n}\right)}\right| .
\end{aligned}
$$


The fact that the functions $x \mapsto g_{i}(x)$ are compactly supported implies that

$$
\sup _{i, k}\left|\int_{E}\left(1-\exp \left(-g_{i}(z) \mathbf{1}_{\left(t_{i}, t_{i+1}\right]}\left(\frac{\tau_{k}}{n}\right)\right)\right) v(\mathrm{~d} z)\right|<C \quad \text { for all } n \in \mathbb{N},
$$

which yields

$$
\left|R_{[n T]} \sup _{i, k}\right| \log \left(\varphi_{i, k ; n}\right) \mid \leq C n^{-1 / \alpha} R_{[n T]} \quad \text { for all } n \in \mathbb{N} \text {. }
$$

Moreover, we know from (R3) that $n^{-1 / \alpha} R_{[n T]}$ converges in distribution to $m(Y(0, T))$. Hence, it follows that

$$
\mathrm{P}\left(\left|R_{[n T]} \log \left(\varphi_{i, k ; n}\right)\right|>N\right) \rightarrow \mathrm{P}(m(Y(0, T))>N) \quad \text { as } n \rightarrow \infty .
$$

Since the right-hand side can be made arbitrarily small by choosing $N \in \mathbb{N}$ large enough, we obtain

$$
\left|\sum_{i, k} \log \left(\lambda_{i, k ; n}\right)-\sum_{i, k} \log \left(\varphi_{i, k ; n}\right)\right| \rightarrow 0 \quad \text { in probability as } n \rightarrow \infty,
$$

which is equivalent to

$$
\left|\prod_{i, k} \lambda_{i, k ; n}-\prod_{i, k} \varphi_{i, k ; n}\right| \rightarrow 0 \quad \text { in probability as } n \rightarrow \infty .
$$

Taking expectations and using dominated convergence shows that the sequence

$$
\mathcal{L}_{n}\left(f_{m}\right)=\mathrm{E}\left[\prod_{k} \prod_{i}\left(1-\int_{E}\left(1-\exp \left(-g_{i}(z) \mathbf{1}_{\left(t_{i}, t_{i+1}\right]}\left(\frac{\tau_{k}}{n}\right)\right)\right) P_{\left[n^{1 / \alpha}\right]}(\mathrm{d} z)\right)\right]
$$

has the same limit as

$$
\begin{aligned}
\mathrm{E}\left[\prod_{k}\right. & \left.\prod_{i} \exp \left(-n^{-1 / \alpha} \int_{E}\left(1-\exp \left(-g_{i}(z) \mathbf{1}_{\left(t_{i}, t_{i+1}\right]}\left(\frac{\tau_{k}}{n}\right)\right)\right) v(\mathrm{~d} z)\right)\right] \\
= & \mathrm{E}\left[\exp \left(-\sum_{k} \sum_{i} n^{-1 / \alpha} \int_{E}\left(1-\exp \left(-g_{i}(z) \mathbf{1}_{\left(t_{i}, t_{i+1}\right]}\left(\frac{\tau_{k}}{n}\right)\right)\right) v(\mathrm{~d} z)\right)\right] \\
= & \mathrm{E}\left[\exp \left(-\sum_{k} \sum_{i} n^{-1 / \alpha} \int_{E}\left(1-\exp \left(-g_{i}(z)\right)\right) \mathbf{1}_{\left(t_{i}, t_{i+1}\right]}\left(\frac{\tau_{k}}{n}\right) v(\mathrm{~d} z)\right)\right] \\
= & \mathrm{E}\left[\exp \left(-\sum_{i} n^{-1 / \alpha} \int_{E}\left(1-\exp \left(-g_{i}(z)\right)\right) v(\mathrm{~d} z)\left(R_{\left[n t_{i+1}\right]}-R_{\left[n t_{i}\right]}\right)\right)\right] .
\end{aligned}
$$

It then follows from (R3) that the previous sequence converges to

$$
\begin{gathered}
\mathrm{E}\left[\exp \left(-\sum_{i} \int_{E}\left(1-\exp \left(-g_{i}(z)\right)\right) v(\mathrm{~d} z)\left(m\left(Y\left(0, t_{i+1}\right)\right)-m\left(Y\left(0, t_{i}\right)\right)\right)\right)\right] \\
=\mathrm{E}\left[\exp \left(-\int_{\mathbb{R}^{+}} \int_{E}\left(1-\exp \left(-f_{m}(z, t)\right)\right) v(\mathrm{~d} z) m_{Y}(\mathrm{~d} t)\right)\right]
\end{gathered}
$$


which is just $\mathcal{L}\left(f_{m}\right)$. Since $f_{m} \rightarrow f$ in the supremum norm as $m \rightarrow \infty$, it follows that

$$
\mathcal{L}_{n}\left(f_{m}\right) \rightarrow \mathcal{L}_{n}(f) \quad \text { as } m \rightarrow \infty
$$

uniformly in $n \in \mathbb{N}$. Moreover, we have just proved that, for all $m \in \mathbb{N}$,

$$
\mathcal{L}_{n}\left(f_{m}\right) \rightarrow \mathcal{L}\left(f_{m}\right) \text { as } n \rightarrow \infty .
$$

Therefore, we have

$$
\mathcal{L}_{n}(f) \rightarrow \mathcal{L}(f) \quad \text { as } n \rightarrow \infty
$$

This proves Theorem 4 .

Proofs of Theorem 1 and Theorem 2. In order to prove Theorem 1, we define the map

$$
\mathfrak{F}: \mathcal{M}_{p}\left(\mathbb{R}^{+} \times E\right) \rightarrow D(0, \infty), \quad N=\sum_{k} \delta_{t_{k}, j_{k}} \mapsto\left(t \mapsto \bigvee_{0<t_{k} \leq t} j_{k}\right) .
$$

It can be proved that $\mathfrak{F}\left(N^{(n)}\right)=K^{(n)}$ (see Resnick (1987, p. 209)). Moreover, $\mathfrak{F}$ is continuous $\mathrm{P}_{N}$-almost surely, where $\mathrm{P}_{N}$ denotes the distribution of the point process $N$ on $\mathcal{M}_{p}\left(\mathbb{R}^{+} \times E\right)$ (see Resnick (1987, p. 214)). The continuous mapping theorem and the previous theorem then imply that $K^{(n)}$ converges to $Z=\mathfrak{F}(N)$.

For Theorem 2, we use the random transformations

$$
\mathfrak{f}: \mathbb{R}^{+} \times E \rightarrow \mathbb{R}^{+} \times E, \quad(t, x) \mapsto(m(Y(0, t)), x) .
$$

If we use the transformation formula for Poisson point processes, we can see that $N_{Y}=\mathfrak{f}(N)$ (see Resnick (1987, p. 134)). With the representation $N=\sum_{k} \delta_{\left(t_{k}, j_{k}\right)}$, this implies that

$$
N_{Y}=\sum_{k} \delta_{\left(m\left(Y\left(0, t_{k}\right)\right), j_{k}\right)}
$$

It then follows that

$$
\mathfrak{F}\left(N_{Y}\right)(t)=\bigvee_{0<m\left(Y\left(0, t_{k}\right)\right) \leq t} j_{k}=Z(m(Y(0, t))) .
$$

\section{The long-range dependence}

As we already mentioned in the introduction, the sequence does not satisfy the weak-mixing conditions introduced by Leadbetter if the underlying random walk is recurrent. We investigate this behaviour in this section.

\subsection{The $D\left(u_{n}\right)$ condition}

The $D\left(u_{n}\right)$ condition is a condition which assures a mixing-type behaviour for the tails of the joint distributions of a stationary sequence of random variables. For a sequence of random variables $\left\{\Xi_{i}, i \in \mathbb{N}\right\}$, we denote its joint distribution function by $F_{i_{1}, \ldots, i_{n}}, 1 \leq i_{1}<\cdots<i_{n}$. Let $\left\{u_{n}, n \in \mathbb{N}\right\}$ be a given sequence of increasing positive real numbers.

Condition 1. A stationary sequence of random variables $\left\{\Xi_{i}, i \in \mathbb{N}\right\}$ satisfies the $D\left(u_{n}\right)$ condition if, for any integers $1 \leq i_{1}<\cdots<i_{p}<j_{1}<\cdots<j_{q} \leq n$ with the property that $j_{1}-i_{p} \geq l$, we have

$$
\left|F_{i_{1}, \ldots, i_{p}, j_{1}, \ldots, j_{q}}\left(u_{n}\right)-F_{i_{1}, \ldots, i_{p}}\left(u_{n}\right) F_{j_{1}, \ldots, j_{q}}\left(u_{n}\right)\right| \leq \alpha_{n, l},
$$

where $\alpha_{n, l} \rightarrow 0$ as $n \rightarrow \infty$ for some sequence $l=o(n)$. 
The $D\left(u_{n}\right)$ condition is used to prove that the limit distribution of the maxima of a stationary sequence is of the same type as that of the independent sequence with the same marginal distributions (see Leadbetter et al. (1983, p. 57)).

Proposition 1. For $\alpha>1$, the stationary sequence $\left\{\xi\left(S_{n}\right), n \in \mathbb{N}\right\}$ does not satisfy the condition $D\left(u_{n}\right)$ with $u_{n}=a_{\left[n^{1 / \alpha}\right]} x+b_{\left[n^{1 / \alpha}\right]}$.

Proof. We first note that, for $u_{n}=a_{\left[n^{1 / \alpha}\right]} x+b_{\left[n^{1 / \alpha}\right]}$, we have

$$
n^{1 / \alpha}\left(1-F\left(u_{n}\right)\right) \rightarrow-\log G(x) \quad \text { as } n \rightarrow \infty .
$$

From this, it follows that, as $n \rightarrow \infty$,

$$
\left(F\left(u_{n}\right)\right)^{n^{1 / \alpha}}=\exp \left(n^{1 / \alpha} \log \left(1-\left(1-F\left(u_{n}\right)\right)\right)\right) \sim \exp \left(-n^{1 / \alpha}\left(1-F\left(u_{n}\right)\right)\right) \rightarrow G(x) .
$$

We know from (R3) that $R^{(n)}:=n^{-1 / \alpha} R_{n}$ converges in distribution to $R:=m(Y(0,1))$. Therefore, there exist random variables $\tilde{R}^{(n)}$ and $\tilde{R}$ with the same distribution as $R^{(n)}$ and $R$, respectively, such that $\tilde{R}^{(n)}$ converges to $\tilde{R}$ almost surely (see Billingsley (1986, p. 343)). It then follows that, P-almost surely,

$$
\left(\left(F\left(u_{n}\right)\right)^{n^{1 / \alpha}}\right)^{\tilde{R}^{(n)}} \rightarrow(G(x))^{\tilde{R}} \quad \text { as } n \rightarrow \infty .
$$

Hence, by dominated convergence and Lemma 1,

$$
\begin{aligned}
F_{1, \ldots, n}\left(u_{n}\right) & =\mathrm{P}\left(\xi\left(S_{1}\right) \leq u_{n}, \ldots, \xi\left(S_{n}\right) \leq u_{n}\right) \\
& =\mathrm{E}\left[\mathrm{P}\left(\xi\left(S_{1}\right) \leq u_{n}, \ldots, \xi\left(S_{n}\right) \leq u_{n} \mid R_{n}\right)\right] \\
& =\mathrm{E}\left[\left(F\left(u_{n}\right)\right)^{R_{n}}\right] \\
& =\mathrm{E}\left[\left(F\left(u_{n}\right)\right)^{n^{1 / \alpha}} \tilde{R}^{(n)}\right]
\end{aligned}
$$

converges to

$$
\mathrm{E}\left[(G(x))^{\tilde{R}}\right]=\mathrm{E}\left[(G(x))^{m(Y(0,1))}\right] .
$$

If $l=o(n)$, we can prove in the same way that

$$
F_{1, \ldots, n, n+l, \ldots, 2 n}\left(u_{n}\right) \rightarrow \mathrm{E}\left[(G(x))^{m(Y(0,2))}\right] \quad \text { as } n \rightarrow \infty
$$

and

$$
F_{n+l, \ldots, 2 n}\left(u_{n}\right)=F_{1, \ldots, n-l}\left(u_{n}\right) \rightarrow \mathrm{E}\left[(G(x))^{m(Y(0,1))}\right] \quad \text { as } n \rightarrow \infty .
$$

It then follows that $\left|F_{1, \ldots, n, n+l, \ldots, 2 n}\left(u_{n}\right)-F_{1, \ldots, n}\left(u_{n}\right) F_{n+l, \ldots, 2 n}\left(u_{n}\right)\right|$ converges to

$$
\begin{aligned}
& \mathrm{E}\left[(G(x))^{m(Y(0,2))}\right]-\left(\mathrm{E}\left[(G(x))^{m(Y(0,1))}\right]\right)^{2} \\
& \quad>\mathrm{E}\left[(G(x))^{m(Y(0,1))+m(Y(1,2))}\right]-\mathrm{E}\left[(G(x))^{m(Y(0,1))}\right]^{2} \\
& \quad=0
\end{aligned}
$$

where we used the fact that $m(Y(0,2))$ is strictly smaller than $m(Y(0,1))+m(Y(1,2))$ almost surely, and the fact that the two random variables $m(Y(0,1))$ and $m(Y(1,2))$ are independent. This proves that the $D\left(u_{n}\right)$ condition does not hold for the sequence $u_{n}=a_{\left[n^{1 / \alpha}\right]} x+b_{\left[n^{1 / \alpha}\right]}$. 
Proposition 2. For $\alpha \leq 1$, the sequence $\left\{\xi\left(S_{n}\right), n \in \mathbb{N}\right\}$ satisfies the $D\left(u_{n}\right)$ condition with

$$
u_{n}:= \begin{cases}a_{[q n]} x+b_{[q n]} & \text { for } \alpha<1, \\ a_{[n / h(n)]} x+b_{[n / h(n)]} & \text { for } \alpha=1 .\end{cases}
$$

Proof. Assume that, for a fixed $\varepsilon>0$, there exists, for every $n \in \mathbb{N}$, a family of integers $1 \leq i_{1}<\cdots<i_{p}<j_{1}<\cdots<j_{q} \leq 2 n$ with the property that $j_{1}-i_{p} \geq l(n)$, where $l(n) / n \rightarrow 0$ as $n \rightarrow \infty$ and

$$
\left|F_{i_{1}, \ldots, i_{p}, j_{1}, \ldots, j_{q}}\left(u_{n}\right)-F_{i_{1}, \ldots, i_{p}}\left(u_{n}\right) F_{j_{1}, \ldots, j_{q}}\left(u_{n}\right)\right|>\varepsilon .
$$

We define $R_{j_{1}, \ldots, j_{q}}:=\operatorname{card}\left\{S_{j_{1}}, \ldots, S_{j_{q}}\right\}, R_{i_{1}, \ldots, i_{p}}:=\operatorname{card}\left\{S_{i_{1}}, \ldots, S_{i_{p}}\right\}$, and

$$
R_{j_{1}, \ldots, j_{q}}^{i_{1}, \ldots, i_{p}}:=R_{j_{1}, \ldots, j_{q}}+R_{i_{1}, \ldots, i_{p}}-\operatorname{card}\left\{S_{i_{1}}, \ldots, S_{i_{p}}, S_{j_{1}}, \ldots, S_{j_{q}}\right\}
$$

It then follows that

$$
\begin{aligned}
& \left(F\left(u_{n}\right)\right)^{\operatorname{card}\left\{S_{i_{1}}, \ldots, S_{i_{p}}, S_{j_{1}}, \ldots, S_{j_{q}}\right\}}-\left(F\left(u_{n}\right)\right)^{R_{j_{1}, \ldots, j_{q}}+R_{i_{1}, \ldots, i_{p}}} \\
& \quad=\left(F\left(u_{n}\right)\right)^{R_{j_{1}, \ldots, j_{q}}+R_{i_{1}, \ldots, i_{p}}}\left(\left(F\left(u_{n}\right)\right)^{\left.-R_{j_{1}, \ldots, j_{q}}^{i_{1}, \ldots, i_{p}}-1\right)}\right. \\
& \quad \leq\left(\left(F\left(u_{n}\right)\right)^{-R_{i_{p}+l, \ldots, 2 n}^{1, \ldots, i_{p}}}-1\right) .
\end{aligned}
$$

We note that $i_{p}$ is a sequence of integers, where the $n$th element is bounded by $2 n$. It follows that $i_{p} / n$ must have convergent subsequences. We can therefore assume without loss of generality that $i_{p} / n \rightarrow u$ as $n \rightarrow \infty$. It follows from (R1) that the sequence

$$
\begin{aligned}
\frac{1}{[n q]} R_{i_{p}+l, \ldots, 2 n}^{1, \ldots, i_{p}} & =\frac{1}{[n q]}\left(R_{1, \ldots, i_{p}}+R_{i_{p}+l, \ldots, 2 n}-R_{1, \ldots, 2 n}\right) \\
& \sim \frac{1}{[n q]}\left(R_{1, \ldots,[n u]}+R_{[n u]+l, \ldots, 2 n}-R_{1, \ldots, 2 n}\right)
\end{aligned}
$$

converges almost surely to $u+(2 n-u)-2 n=0$ as $n \rightarrow \infty$. As we have

$$
q n\left(1-F\left(u_{n}\right)\right) \rightarrow-\log G(x) \text { as } n \rightarrow \infty,
$$

it follows that

$$
\left(F\left(u_{n}\right)\right)^{n q} \sim \exp \left(-n q\left(1-F\left(u_{n}\right)\right)\right) \rightarrow G(x) \text { as } n \rightarrow \infty .
$$

This together with the convergence of $(1 /[n q]) R_{i_{p}+l, \ldots, 2 n}^{1, \ldots, i_{p}}$ toward 0 implies that, P-almost surely,

$$
\left(\left(F\left(u_{n}\right)\right)^{\left.-R_{i p}^{1, \ldots, i_{p}, \ldots, 2 n}-1\right) \rightarrow 0 \quad \text { as } n \rightarrow \infty .}\right.
$$

We note that

$$
\begin{aligned}
F_{j_{1}, \ldots, j_{q}}\left(u_{n}\right) F_{i_{1}, \ldots, i_{p}}\left(u_{n}\right) \\
=\mathrm{E}\left[\left(F\left(u_{n}\right)\right)^{R_{j_{1}, \ldots, j_{q}}}\right] \mathrm{E}\left[\left(F\left(u_{n}\right)\right)^{R_{i_{1}, \ldots, i_{p}}}\right] \\
=\mathrm{E}\left[\mathrm{E}\left[\left(F\left(u_{n}\right)\right)^{R_{j_{1}, \ldots, j_{q}}} \mid S_{i_{1}}, \ldots, S_{i_{p}}\right]\left(F\left(u_{n}\right)\right)^{R_{i_{1}, \ldots, i_{p}}}\right] \\
=\mathrm{E}\left[\mathrm{E}\left[\left(F\left(u_{n}\right)\right)^{R_{j_{1}, \ldots, j_{q}}}\left(F\left(u_{n}\right)\right)^{R_{i_{1}, \ldots, i_{p}}} \mid S_{i_{1}}, \ldots, S_{i_{p}}\right]\right] \\
=\mathrm{E}\left[\left(F\left(u_{n}\right)\right)^{R_{j_{1}, \ldots, j_{q}}}\left(F\left(u_{n}\right)\right)^{R_{i_{1}, \ldots, i_{p}}}\right] .
\end{aligned}
$$


This together with (2) and (3) implies that

$$
\begin{aligned}
& \left|F_{i_{1}, \ldots, i_{p}, j_{1}, \ldots, j_{q}}\left(u_{n}\right)-F_{i_{1}, \ldots, i_{p}}\left(u_{n}\right) F_{j_{1}, \ldots, j_{q}}\left(u_{n}\right)\right| \\
& \quad=\mathrm{E}\left[\left(F\left(u_{n}\right)\right)^{\mathrm{card}\left\{S_{i_{1}}, \ldots, S_{i_{p}}, S_{j_{1}}, \ldots, S_{j_{q}}\right\}}-\left(F\left(u_{n}\right)\right)^{\left.R_{j_{1}, \ldots, j_{q}}+R_{i_{1}, \ldots, i_{p}}\right]}\right. \\
& \quad \leq \mathrm{E}\left[\left(\left(F\left(u_{n}\right)\right)^{\left.\left.-R_{i_{p}+l, \ldots, 2 n}^{1, \ldots, i_{p}}-1\right)\right]}\right.\right.
\end{aligned}
$$

converges to 0 . This contradicts the initial assumption and proves the first statement of the proposition. The second statement follows in the same way by using (R2) instead of (R1).

\subsection{The $D^{\prime}\left(u_{n}\right)$ condition for $\alpha<1$}

The following condition is called the $D^{\prime}\left(u_{n}\right)$ condition in the literature.

Condition 2. A stationary sequence of random variables $\left\{\Xi_{i}, i \in \mathbb{N}\right\}$ satisfies the $D^{\prime}\left(u_{n}\right)$ condition if

$$
\limsup _{n \rightarrow \infty} n \sum_{j=2}^{[n / k]} \mathrm{P}\left(\Xi_{1}>u_{n}, \Xi_{j}>u_{n}\right) \rightarrow 0 \quad \text { as } k \rightarrow \infty .
$$

The $D^{\prime}\left(u_{n}\right)$ condition together with the $D\left(u_{n}\right)$ condition implies that

$$
\mathrm{P}\left(\max \left\{\Xi_{1}, \ldots, \Xi_{n}\right\} \leq u_{n}\right)-\mathrm{P}\left(\max \left\{\tilde{\Xi}_{1}, \ldots, \tilde{\Xi}_{n}\right\} \leq u_{n}\right) \rightarrow 0 \quad \text { as } n \rightarrow \infty,
$$

where $\left\{\tilde{\Xi}_{n}, n \in \mathbb{N}\right\}$ is an independent sequence with the same marginals as $\left\{\Xi_{n}, n \in \mathbb{N}\right\}$ (see Leadbetter et al. (1983, p. 61)).

Proposition 3. For $\alpha<1$, the sequence $\left\{\xi\left(S_{n}\right), n \in \mathbb{N}\right\}$ does not satisfy the $D^{\prime}\left(u_{n}\right)$ condition with $u_{n}=a_{[q n]} x+b_{[q n]}$.

Proof. Owing to the definition of the sequences $u_{n}$, we have

$$
q n\left(1-F\left(u_{n}\right)\right) \rightarrow-\log (G(x)) \quad \text { as } n \rightarrow \infty .
$$

We have

$$
\begin{aligned}
n \sum_{j=1}^{[n / k]} \mathrm{P}\left(\xi\left(S_{1}\right)>u_{n}, \xi\left(S_{j}\right)>u_{n}\right) \\
=n \sum_{j=1}^{[n / k]} \mathrm{P}\left(\xi\left(S_{1}\right)>u_{n}, \xi\left(S_{j}\right)>u_{n} \mid S_{j}=S_{1}\right) \mathrm{P}\left(S_{j}=S_{1}\right) \\
\quad+n \sum_{j=1}^{[n / k]} \mathrm{P}\left(\xi\left(S_{1}\right)>u_{n}, \xi\left(S_{j}\right)>u_{n} \mid S_{j} \neq S_{1}\right) \mathrm{P}\left(S_{j} \neq S_{1}\right) \\
=n\left(1-F\left(u_{n}\right)\right) \sum_{j=1}^{[n / k]} \mathrm{P}\left(S_{j}=S_{1}\right)+n\left(1-F\left(u_{n}\right)\right)^{2} \sum_{j=1}^{[n / k]} \mathrm{P}\left(S_{j} \neq S_{1}\right)
\end{aligned}
$$

Since we have

$$
0 \leq \sum_{j=1}^{[n / k]} \mathrm{P}\left(S_{j} \neq S_{1}\right) \leq\left[\frac{n}{k}\right]
$$


it follows for the second term on the right-hand side of (4) that

$$
\lim _{k \rightarrow \infty} \lim _{n \rightarrow \infty} n\left(1-F\left(u_{n}\right)\right)^{2} \sum_{j=1}^{[n / k]} \mathrm{P}\left(S_{j} \neq S_{1}\right)=-\lim _{k \rightarrow \infty} \frac{\log (G(x))}{k}=0 .
$$

It follows for the first term on the right-hand side of (4) that

$$
\begin{aligned}
\lim _{n \rightarrow \infty} n\left(1-F\left(u_{n}\right)\right) \sum_{j=1}^{[n / k]} \mathrm{P}\left(S_{j}=S_{1}\right) & \geq-\log (G(x)) \mathrm{P}\left(\text { there exists } j \in \mathbb{N}: S_{j}=S_{1}\right) \\
& =\log (G(x))(1-q) .
\end{aligned}
$$

This is larger than 0 since, for $\alpha<1$, we have $0<q<1$.

Remark. Proposition 3 also follows from the fact that the extremal index is $q$ (see Leadbetter (1983, p. 59)).

\subsection{The extremal index}

The extremal index is a measure for the dependence in the tails of a stationary sequence. This concept appeared in the work of Newell (1964) and Loynes (1965) for $m$-dependent variables and was later named the extremal index in Leadbetter (1983).

Definition. A $\theta \in[0,1]$ is called an extremal index for the stationary sequence $\left\{\Xi_{i}, i \in \mathbb{N}\right\}$ if, for every $\tau>0$,

(i) there exists a sequence $v_{n} \uparrow \infty$ such that $n\left(1-F\left(v_{n}\right)\right) \rightarrow \tau$,

(ii) $\mathrm{P}\left(\max \left\{\Xi_{1}, \ldots, \Xi_{n}\right\} \leq v_{n}\right) \rightarrow \mathrm{e}^{-\tau \theta}$.

The extremal index is an indicator for the existence of clusters of exceedances (see Hsing et al. (1988)). Usually, for a model with extremal index $\theta \in(0,1)$, the observed cluster size is $1 / \theta$. In our situation the expected number of visits to a site is $1 / q$. This motivates the following proposition.

Proposition 4. The extremal index of the sequence $\left\{\xi\left(S_{n}\right), n \in \mathbb{N}\right\}$ is equal to $q=\mathrm{P}\left(S_{k} \neq 0\right.$, $k \in \mathbb{N})$.

Proof. Let $\left\{v_{n}, n \in \mathbb{N}\right\}$ be a sequence such that

$$
n\left(1-F\left(v_{n}\right)\right) \rightarrow \tau \quad \text { as } n \rightarrow \infty .
$$

We know from the theorem of Kesten, Spitzer, and Whitman that $R_{n} / n$ converges almost surely to $q=\mathrm{P}\left(S_{k} \neq 0, k \in \mathbb{N}\right)$ as $n \rightarrow \infty$ (see Spitzer (1976, p. 38)). Then it follows from Lemma 1 that

$$
\begin{aligned}
\mathrm{P}\left(\max \left\{\xi\left(S_{1}\right), \ldots, \xi\left(S_{n}\right)\right\} \leq v_{n}\right) & =\mathrm{P}\left(\xi\left(S_{1}\right) \leq v_{n}, \ldots, \xi\left(S_{n}\right) \leq v_{n}\right) \\
& =\sum_{k=1}^{n} \mathrm{P}\left(\xi\left(S_{\tau_{1}}\right) \leq v_{n}, \ldots, \xi\left(S_{\tau_{k}}\right) \leq v_{n}, R_{n}=k\right) \\
& =\sum_{k=1}^{n} \mathrm{P}\left(\xi(1) \leq v_{n}, \ldots, \xi(k) \leq v_{n}\right) \mathrm{P}\left(R_{n}=k\right) \\
& =\mathrm{E}\left[\left(F\left(v_{n}\right)\right)^{R_{n}}\right]
\end{aligned}
$$


We note that by the assumption on the sequence $\left\{v_{n}, n \in \mathbb{N}\right\}$ as $n \rightarrow \infty$ we have

$$
\left(F\left(v_{n}\right)\right)^{n}=\exp \left(n \log \left(1-\left(1-F\left(v_{n}\right)\right)\right)\right) \sim \exp \left(-n\left(1-F\left(v_{n}\right)\right)\right) \rightarrow \mathrm{e}^{-\tau} .
$$

This implies, together with the result of Kesten, Spitzer, and Whitman, that, P-almost surely,

$$
\left(F\left(v_{n}\right)\right)^{R_{n}}=\left(\left(F\left(v_{n}\right)\right)^{n}\right)^{R_{n} / n} \rightarrow \mathrm{e}^{-q \tau} \quad \text { as } n \rightarrow \infty .
$$

Hence, by dominated convergence we have

$$
\mathrm{P}\left(\max \left\{\xi\left(S_{1}\right), \ldots, \xi\left(S_{n}\right)\right\} \leq v_{n}\right)=\mathrm{E}\left[\left(F\left(v_{n}\right)\right)^{R_{n}}\right] \rightarrow \mathrm{e}^{-q \tau} \quad \text { as } n \rightarrow \infty .
$$

This proves that the extremal index is $q$.

Remark. We note that $q=0$ is equivalent to the random walk being recurrent, i.e. $\alpha>1$.

\subsection{The type of limit distribution for $\alpha>1$}

It is of course interesting to determine whether in the recurrent case the limit distribution has one of the extremal types. This is, however, not the case.

Proposition 5. The distribution $H$ of $Z(m(Y(0,1)))$ is not an extreme value distribution.

Proof. If $G$ is the distribution function associated with the extreme value process $Z$, we have

$$
H(x)=\mathrm{P}(Z(m(Y(0,1))) \leq x)=\mathrm{E}\left[(G(x))^{m(Y(0,1))}\right] .
$$

We first concentrate on the case where $\{\xi(k), k \in \mathbb{Z}\}$ is in the domain of attraction of a Fréchet distribution $\Phi_{\gamma}$. We then have, for all $x \geq 0$,

$$
H(x)=\mathrm{E}\left[\left(\Phi_{\gamma}(x)\right)^{m(Y(0,1))}\right]=\int_{0}^{\infty} \exp \left(-t x^{-\gamma}\right) \mathrm{P}_{m(Y(0,1))}(\mathrm{d} t) .
$$

From this expression we see that the support of $H$ is equal to $[0, \infty)$. If we assume that $H$ is an extreme value distribution, it must be a Fréchet distribution $\Phi_{\gamma^{\prime}}$. Furthermore, it is easy to see from the previous expression that $1-H$ is regularly varying with exponent $\gamma$. This implies that $\gamma^{\prime}=\gamma$ and that $H(x)$ is equal to $\Phi_{\gamma}(a x-b)$ for suitable $a>0$ and $b \in \mathbb{R}$. Since the support of $H$ is $[0, \infty)$, it follows that $b=0$. Moreover, we can see from the previous equation that

$$
\mathrm{e}^{-a x}=H\left(x^{\gamma}\right)=\int_{0}^{\infty} \mathrm{e}^{-t x} \mathrm{P}_{m(Y(0,1))}(\mathrm{d} t),
$$

which is the Laplace transform of the distribution of $m(Y(0,1))$. This is a contradiction, since this would imply that $m(Y(0,1))$ is constant. Therefore, $H$ cannot be an extreme value distribution.

A similar reasoning works if $\{\xi(k), k \in \mathbb{Z}\}$ is in the domain of attraction of a Weibull distribution $\Psi_{\gamma}$. Again, we assume that $H$ is an extreme value distribution. Comparison of the support shows that $H$ must be a Weibull distribution $\Psi_{\gamma^{\prime}}$. A regular variation argument shows that $\gamma^{\prime}=\gamma$. A change of variable unveils an equality involving the Laplace transform of $m(Y(0,1))$, which would imply that $m(Y(0,1))$ is constant. This is obviously wrong and, therefore, $H$ cannot be an extreme value distribution.

If $\{\xi(k), k \in \mathbb{Z}\}$ is in the domain of attraction of a Gumbel distribution, and if $H$ was an extreme value distribution, then it could only be a Gumbel distribution. This follows from the fact that $H$ has full support on $\mathbb{R}$. A suitable change of variables again leads to an equation involving the Laplace transform of $m(Y(0,1))$. The expression for the Laplace transform would imply that $m(Y(0,1))$ is constant, which is not true. 


\subsection{Chain-dependent Markov processes}

Chain-dependent Markov processes are special stationary sequences, where the dependence comes from an underlying Markovian structure. Their extreme value behaviour has been studied extensively in the past three decades (see Resnick (1972), Denzel and O'Brien (1975), Turkman and Oliveira (1992), and Fereira (1998)). It is worth mentioning that applications of chaindependent processes to meteorology have been described in Katz (1977). In the following, let $\mathcal{Z}$ be a countable set.

Definition. A double sequence of random variables $\left\{\Xi_{n}, \zeta_{n}, n \in \mathbb{N}\right\}$ is called a chain-dependent Markov process with state space $\mathbb{R} \times \mathbb{Z}$ if, for all $i, j \in \mathcal{Z}$ and $x \in \mathbb{R}$,

$$
\mathrm{P}\left(\Xi_{n} \leq x, \zeta_{n}=j \mid \Xi_{0}, \zeta_{0}, \ldots, \Xi_{n-1}, \zeta_{n-1}=i\right)=\mathrm{P}\left(\Xi_{n} \leq x, \zeta_{n}=j \mid \zeta_{n-1}=i\right),
$$

where the right-hand side does not depend on $n$.

Proposition 6. The double sequence $\left\{\xi\left(S_{n}\right), S_{n}, n \in \mathbb{N}\right\}$ is not a chain-dependent Markov process.

Proof. Let $i$ and $j$ be integers such that $\mathrm{P}\left(S_{n}=j \mid S_{n-1}=i\right) \neq 0$. Then we have

$$
\begin{aligned}
& 0=\mathrm{P}\left(\xi\left(S_{n}\right) \leq x, S_{n}=j \mid \xi\left(S_{1}\right)>x, S_{1}=j, S_{n-1}=i\right) \\
& \quad \neq \mathrm{P}\left(\xi\left(S_{n}\right) \leq x, S_{n}=j \mid S_{n-1}=i\right) .
\end{aligned}
$$

This completes the proof.

A generalisation of chain-dependent stationary sequences was described in Turkman and Walker (1983). The process $\left\{\xi\left(S_{n}\right), S_{n}, n \in \mathbb{N}\right\}$ satisfies some of the structural properties of the sequences $\left\{\Xi_{n}, \zeta_{n}, n \in \mathbb{N}\right\}$ considered there. It satisfies the $\zeta_{n}$-dependence of $\Xi_{n}$, i.e.

$$
\mathrm{P}\left(\Xi_{n} \leq x \mid \zeta_{1}=s_{1}, \ldots, \zeta_{n}=s_{n}\right)=\mathrm{P}\left(\Xi_{n} \leq x \mid \zeta_{n}=s_{n}\right)
$$

and the conditional independence of $\Xi_{1}, \ldots, \Xi_{n}$ from $\left\{S_{n+k}, k \in \mathbb{N}\right\}$, i.e.

$$
\begin{aligned}
& \mathrm{P}\left(\Xi_{1} \leq x_{1}, \ldots, \Xi_{n} \leq x_{n} \mid \zeta_{1}=s_{1}, \ldots, \zeta_{n+k}=s_{n+k}\right) \\
& \quad=\mathrm{P}\left(\Xi_{1} \leq x_{1}, \ldots, \Xi_{n} \leq x_{n} \mid \zeta_{1}=s_{1}, \ldots, \zeta_{n}=s_{n}\right) .
\end{aligned}
$$

However, they only considered sequences of random variables $\left\{\zeta_{n}, n \in \mathbb{N}\right\}$ with a finite state space $\mathcal{Z}$. This is not the case in our situation.

\section{Acknowledgements}

The authors want to express their gratitude to Narn-Rueih Shieh for many interesting discussions on the subject of this article and Wolfgang König for some communication on the paper of Kesten and Spitzer. Moreover, we want to thank Huai-Yao Huang for proofreading this manuscript. The first author wants to thank Academia Sinica for financial support. The second author was supported by a postdoctoral grant from Taiwan NSC. We also want to thank Patrik Albin for some helpful comments on a first version of this manuscript. 


\section{References}

AraI, T. (2001). A class of semi-selfsimilar processes related to random walks in random scenery. Tokyo J. Math. 24, 69-85.

Billingsley, P. (1986). Probability and Measure, 2nd edn. John Wiley, New York.

Daley, D. J. And Vere-Jones, D. (1988). An Introduction to the Theory of Point Processes. Springer, New York.

Denzel, G. E. And O'Brien, G. (1975). Limit theorems for extreme values of chain-dependent processes. Ann. Prob. 3, 773-779.

Fereira, H. (1998). Doubly stochastic compound Poisson processes in extreme value theory. Portugal. Math. 55, 465-474.

Gnedenko, B. (1943). Sur la distribution limite du terme d'une série aléatoire. Ann. Math. 44, 423-453.

Hsing, T., Hüsler, J. and LeadbetTer, M. R. (1988). On the exceedance point process for a stationary sequence. Prob. Theory Relat. Fields 78, 97-112.

Katz, R. W. (1977). An application of chain-dependent processes to meteorology. J. Appl. Prob. 14, $598-603$.

Kesten, H. And SpITZER, F. (1979). A limit theorem related to a new class of self-similar processes. Z. Wahrscheinlichkeitsth. 50, 5-25.

LANG, R. AND NGUYEN, X.-X. (1983). Strongly correlated random fields as observed by a random walker. Z. Wahrscheinlichkeitsth. 64, 327-340.

Leadbetter, M. R. (1983). Extremes and local dependence in stationary sequences. Z. Wahrscheinlichkeitsth., 65, 291-306.

Leadbetter, M. R., Lindgren, G. And Rootzén, H. (1983). Extremes and Related Properties of Random Sequences and Processes. Springer, New York.

Le Gall, J.-F. And Rosen, J. (1991). The range of stable random walks. Ann. Prob. 19, 650-705.

Loynes, R. M. (1965). Extreme values in uniformly mixing stationary stochastic processes. Ann. Math. Statist. 36, 993-999.

Maejima, M. (1996). Limit theorems related to a class of operator-self-similar processes. Nagoya Math. J. 142, 161181.

Newell, G. F. (1964). Asymptotic extremes for $m$-dependent random variables. Ann. Math. Statist. 35, $1322-1325$.

ReSNick, S. I. (1972). Stability of maxima of random variables defined on a Markov chain. Adv. Appl. Prob. 4, $285-295$.

Resnick, S. I. (1987). Extreme Values, Regular Variation, and Point Processes (Appl. Prob. 4). Springer, New York.

SAIGO, T. AND TAKAHASHI, H. (2005). Limit theorems related to a class of operator semi-selfsimilar processes. J. Math. Sci. Univ. Tokyo 12, 111-140.

Shieh, N. R. (1995). Some self-similar processes related to local times. Statist. Prob. Lett. 24, 213-218.

SpITZER, F. (1976). Principles of Random Walks, 2nd edn. Springer, New York.

Turkman, K. F. And Oliveira, M. F. (1992). Limit laws for the maxima of chain-dependent sequences with positive extremal index. J. Appl. Prob. 29, 222-227.

Turkman, K. F. AND WAlker, A. M. (1983). Limit laws for the maxima of a class of quasistationary sequences. J. Appl. Prob. 20, 814-821. 